\title{
The Far Infrared Spectrum of Vulcanized Natural Rubber
}

\author{
James E. Stewart* and Frederic J. Linnig \\ Institute for Materials Research, National Bureau of Standards, Washington, D.C., 20234
}

(August 15. 1966)

\begin{abstract}
Earlier studies of the infrared spectra of natural rubber and some vulcanizates of rubber have been extended into the 650 to $33 \mathrm{~cm}^{-1}$ far infrared region. Some of the absorption bands originating in the crystalline portion of unvulcanized rubber have been studied by using polarized radiation and samples oriented by stretching. Vulcanization of rubber with sulfur alone produces a general weakening of all absorption bands and no new absorptions. Vulcanization with sulfur and an accelerator also results in a reduction in intensity of the rubber absorptions, but is accompanied by the appearance of new bands. When the accelerator is tetramethylthiuram disulfide (TMTD) the new bands are probably indicative of a thiocarbamate structure. When zinc dibutyldithiocarbamate is used as an accelerator a band probably due to zinc sulfide occurs. Vulcanization with TMTD alone produces only slight changes in the far infrared spectrum. However, when TMTD is used in the presence of zinc oxide a reaction occurs in which zinc dimethyldithiocarbamate (ZnDMDC) is formed. After vulcanization for a short period of time strong TMTD bands remain, but after long vulcanization the TMTD bands are replaced by ZnDMDC bands.
\end{abstract}

Key Words: Accelerators, far infrared, natural rubber, sulfur, tetramethylthiuram disulfide, vulcanization, zinc dibutyldithiocarbamate, zinc dimethyldithiocarbamate.

\section{Introduction}

In previous reports $[1,2]^{1}$ we discussed the absorption spectra of natural rubber, its vulcanizates, and of squalene as a model compound in the ultraviolet, visible, near infrared, and mid infrared. The far infrared has only recently become available on anything like a routine basis. We have extended our work into this region in order to investigate the usefulness of the far infrared in vulcanization studies. In this article we shall discuss the low-frequency infrared spectra of natural rubber and some of its vulcanizates in the 650 to $33 \mathrm{~cm}^{-1}$ region.

\section{Experimental Procedure}

Unvulcanized samples were prepared from natural rubber by milling it at $70{ }^{\circ} \mathrm{C}$ for $15 \mathrm{~min}$ and pressing it between sheets of Teflon for $7 \mathrm{~min}$ at 100 to $125^{\circ} \mathrm{C}$. Attempts to prepare films of unmilled rubber were not successful.

Vulcanized samples were prepared between sheets of aluminum foil in the manner described previously $[1,2]$. The compositions of the vulcanized samples are tabulated in table 1. Except where noted in the figures, all samples were approximately $0.2 \mathrm{~mm}$ thick.

* Present address: Beckman Instruments, Inc., Fulierton, Calif., 92634.

' Figures in brackets indicate the literature references at the end of this paper.
In every case, natural rubber NBS Standard Sample No. 385a was used, and in addition, Samples I, II, and III were duplicated with high purity natural rubber No. 2103 of the United States Rubber Company. Other compounding materials were Sulfur (NBS Standard Sample 371d), Stearic Acid (NBS Standard Sample 372e), Zinc Oxide (NBS Standard Sample 370B), Tetramethylthiuram Disulfide or TMTD (NBS Standdard Sample 374b), and Zinc Dibutyldithiocarbamate or ZnDBDC (R. T. Vanderbilt Co.). In addition, samples of Zinc Dimethyldithiocarbamate or ZnDMDC (R. T. Vanderbilt Co.), Squalene (Eastman Kodak Co.), and a squalene-sulfur reaction product were studied.

TABLE 1. Compositions of rubber vulcanizates.

\begin{tabular}{|c|c|c|c|c|c|c|c|c|}
\hline \multirow{2}{*}{ Sample } & \multicolumn{2}{|c|}{ Vulcanization } & \multirow{2}{*}{ Rubber } & \multirow{2}{*}{ Sulfur } & \multirow{2}{*}{$\begin{array}{c}\text { Stearic } \\
\text { acid }\end{array}$} & \multirow{2}{*}{$\begin{array}{c}\text { Zinc } \\
\text { oxide }\end{array}$} & \multirow{2}{*}{ TMTD } & \multirow{2}{*}{$\mathrm{ZnDBDC}$} \\
\hline & Time & Temp. & & & & & & \\
\hline I & $h_{20}$ & $\begin{array}{l}{ }^{\circ} \mathrm{C} \\
150\end{array}$ & $\begin{array}{l}\% \\
97.1\end{array}$ & $\begin{array}{l}\% \\
2.9\end{array}$ & $\%$ & $\%$ & $\%$ & $\%$ \\
\hline II & 1 & 115 & 96.6 & 1.9 & 0.5 & 0.5 & & 0.0 \\
\hline III & $3 / 4$ & 125 & 96.1 & & .5 & .5 & 2.9 & . \\
\hline IV & 70 & 125 & 85.0 & 15.0 & & & & \\
\hline V & 43 & 125 & 77.5 & 15.0 & & ...... & 7.5 & $\ldots \ldots$ \\
\hline VI & 3 & 125 & 77.5 & 15.0 & & & & 7.5 \\
\hline VII & 40 & 125 & 85.0 & & & & 15.0 & \\
\hline VIII & $3 / 4$ & 125 & 78.0 & ...... & 2.0 & 5.0 & 15.0 & $\ldots \ldots$ \\
\hline IX & 20 & 125 & 78.0 & ..... & 2.0 & 5.0 & 15.0 & …............. \\
\hline
\end{tabular}




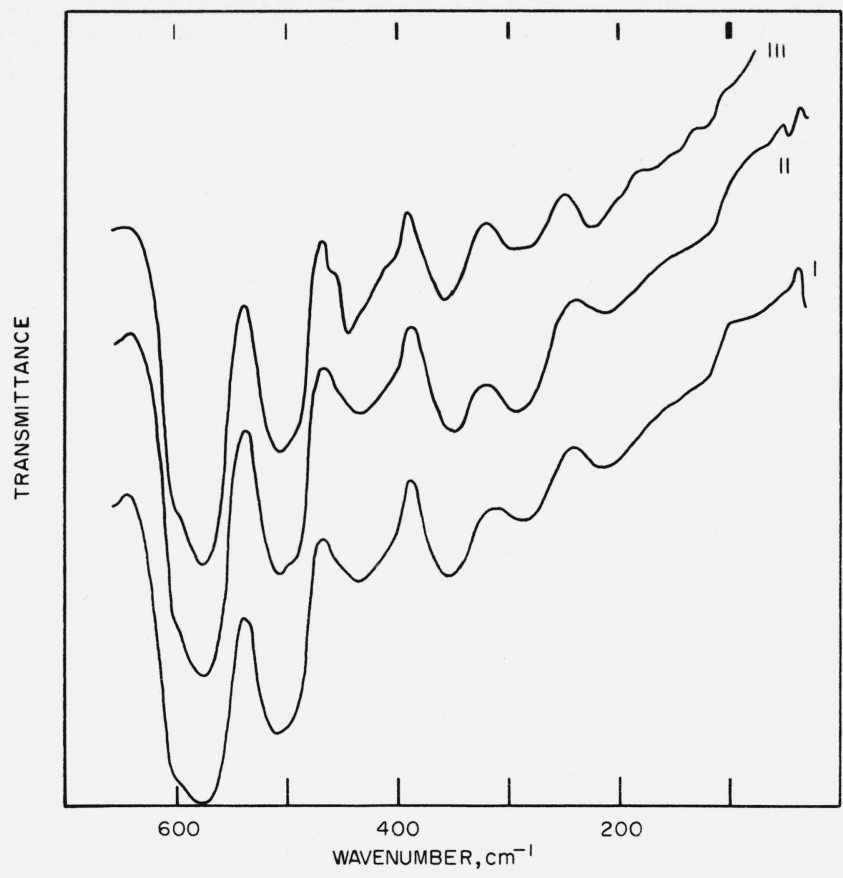

FIGURE 1. Far infrared spectra of natural rubber vulcanized with (I) sulfur; (II) sulfur, ZnDBDC, zinc oxide, and stearic acid; and (III) TMTD, zinc oxide, and stearic acid.

See table 1 for composition, times, and temperatures. Width of lines at the top of the figure indicates the spectral slit width.

Spectra in the 650 to $33 \mathrm{~cm}^{-1}$ region were recorded with a Beckman IR-11.2 Spectral slit widths (reciprocal dispersion multiplied by slit width) between 1 and $5 \mathrm{~cm}^{-1}$ were used throughout the far infrared region. This is quite adequate resolution for the broad absorption bands of rubber. The change in spectral slit width with frequency for all figures except figure 2 is given in figure 1. Stray radiation amounts to about 4 percent or less at $80 \mathrm{~cm}^{-1}, 2$ to 3 percent between 120 and $150 \mathrm{~cm}^{-1}$, and less than 0.4 percent at frequencies higher than $200 \mathrm{~cm}^{-1}$. Studies of oriented samples using polarized radiation were made with a Beckman IR-7. The latter observations were confined to the 650 to $200 \mathrm{~cm}^{-1}$ region by the cesium iodide fore prism. The polarizer consists of ten polyethylene sheets mounted at the Brewster angle. The calculated percentage polarization $\left(I_{p}-I_{s}\right) /\left(I_{p}+I_{s}\right)$, is about 60 percent, in satisfactory agreement with observations using crossed polarizers.

\section{Far Infrared Spectrum of Natural Rubber}

A goal of infrared spectroscopy is to observe and measure absorption bands and to determine the nature of the molecular vibrations which are responsible for

${ }^{2}$ Certain commercial materials and equipment are identified in this paper in order to adquately specify the experimental procedure. In no case does such identification imply recommendation or endorsement by the National Bureau of Standards, nor does it imply recommendation or endorsement by the National Bureau of Standards, nor does it imply
that the material or equipment identified is necessarily the best available for the purpose. them. The low frequency. vibrations of hydrocarbons seen in far infrared spectra are skeletal in nature, involving the entire molecule to some extent. They cannot be considered as localized in any small functional group, as is often the case with vibrations of higher frequency. Nevertheless we wish to characterize them as fully as possible. To this end, we employed the technique of polarized infrared spectroscopy. We have also compared the infrared spectra of other compounds whose structures resemble natural rubber.

In one of the previous studies of natural rubber we observed four prominent bands in the infrared spectrum-at 570 with a shoulder at 590,510 with a shoulder at 490,430 , and $360 \mathrm{~cm}^{-1}$. With the extension of the spectrum to $33 \mathrm{~cm}^{-1}$, additional bands were found at 295, 220, and $120 \mathrm{~cm}^{-1}$. Representative spectra are shown in figure 1 . The absorption bands are broad, with half-widths in the neighborhood of 30 $\mathrm{cm}^{-1}$, and show evidence of overlapping structure. The materials used in obtaining these spectra were vulcanized to some extent. The samples were compounded in different ways (see table 1)-(I), with sulfur alone, (II) with sulfur and an accelerator, ZnDBDC, and (III) with TMTD and no sulfur. The spectra are very similar, except for some small differences which will be discussed. in later sections. The spectrum of unvulcanized natural rubber is indistinguishable from the spectrum of I. Spectra were obtained using purified rubber because some differences had been observed in the mid infrared, particularly around 1600 $\mathrm{cm}^{-1}$, depending on the rubber used. No differences were seen in the far infrared spectra.

Natural rubber contains amorphous and crystalline regions. The proportion of crystalline structure can be enhanced by lowering the temperature or by mechanical elongation. Certain bands in the mid infrared are known to be sensitive to the degree of crystallinity, and in the case of orientation by stretching, the same bands are most responsive to changes of the plane of polarization of the incident radiation $[3,4]$. The polarized infrared spectrum of an oriented film of natural rubber, vulcanized with TMTD and no sulfur, (III) is shown in figure 2. The film was oriented by stretching to about six times its original length. More highly vulcanized samples could not be oriented by stretching, presumably because of the network of cross-links. Unvulcanized rubber on the other hand is difficult to handle. The spectrum of the unstretched sample included in figure 2 is seen to differ somewhat from the spectrum of III in figure 1 , notably in the contrast of the shoulders at 462 and $407 \mathrm{~cm}^{-1}$. The two samples were prepared in the same way, but at different times. The spectral differences might reflect differences in extent of vulcanization. A sample of rubber vulcanized with sulfur alone (I) displayed qualitatively similar dichroic behavior, but not as pronounced as the TMTD vulcanizate. The vibrations responsible for the bands at 570 and $295 \mathrm{~cm}^{-1}$ are found to have their transition moments predominantly parallel to the direction of stretch and therefore 


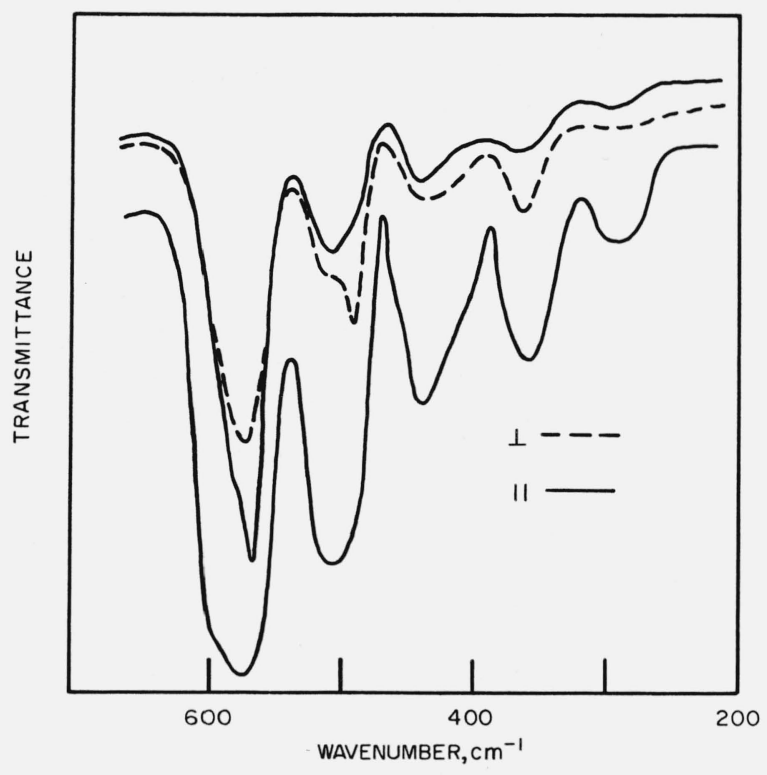

FIGURE 2. Rubber vulcanized with TMTD, zinc oxide and stearic acid (III).

Upper curves: sample oriented by stretching and polarized incident radiation with E-vector parallel and perpendicular to direction of stretch. Thickness not known.

Lower curve: unoriented sample.

parallel to the axis of the polyisoprene chain. The 490 and $360 \mathrm{~cm}^{-1}$ vibrations are predominantly perpendicular. The 570 and $490 \mathrm{~cm}^{-1}$ bands are known to originate in the crystalline portion of the rubber, as demonstrated by low temperature experiments [4]. The shoulders on the high frequency sides of these bands at roughly 590 and $510 \mathrm{~cm}^{-1}$ are not sensitive to polarization and are due to the amorphous portion.

The basic unit of the polyisoprene chain of natural rubber has no symmetry, the conformation of the chain structure is not known, and detailed band assignments of similar molecules are not available. It would be presumptuous to attempt to assign the far infrared absorption bands of rubber to specific vibrations. We can, however, make some observations on the probable nature of some of these bands.

Hydrocarbons containing the $\mathbf{R R}^{\prime}=\mathrm{CHR}^{\prime \prime}$ structure appear to have characteristic strong absorptions at $543 \pm 26$ and $497 \pm 28 \mathrm{~cm}^{-1}$ and usually weaker bands at $437 \pm 25 \mathrm{~cm}^{-1}$ [5]. These vibrations are probably similar in form to those responsible for the 570,500 , and $430 \mathrm{~cm}^{-1}$ absorptions in rubber. Hence, it is reasonable to suppose that the $543 \mathrm{~cm}^{-1}$ vibrations of hydrocarbons are parellel to the $\mathrm{C}=\mathrm{C}$ axis and the $497 \mathrm{~cm}^{-1}$ vibrations are perpendicular.

Shipman and Golub [6] have recently published spectra of polyisoprene, and the deuterated forms polyisoprene $-3-d_{1}$ and polyisoprene $-d_{8}$. In the completely deuterated material the 570 and $490 \mathrm{~cm}^{-1}$ bands are shifted to about 500 and $440 \mathrm{~cm}^{-1}$, very nearly in the ratio of the square roots of the masses of $\mathrm{CD}_{3}$ and $\mathrm{CH}_{3}$ groups. In the singly deuterated polymer the shift is only about $10 \mathrm{~cm}^{-1}$. This is consistent with a surmise that these vibrations are dominated by the methyl group. The far infrared spectrum of polypropylene displays a sequence of bands progressing from 575 to $104 \mathrm{~cm}^{-1}$ and resembling the bands in the rubber spectrum, although weaker by nearly an order of magnitude. The normal coordinate analyses of Miyazawa et al. [7], and of Tadokoro et al. [8], show that $\mathrm{C}-\mathrm{C}-\mathrm{CH}_{3}$ bending motion tends to prevail at the higher frequency end of this range while the $\mathrm{C}-\mathrm{C}-\mathrm{C}$ bending is more important at the lower frequencies.

\section{Sulfur Vulcanizates}

The far infrared spectrum of a sample of natural rubber vulcanized with a small amount of sulfur (I) is included in figure 1 . As previously indicated, it is qualitatively identical to the spectrum of unvulcanized rubber. The spectrum of a sample highly vulcanized with sulfur alone (IV) is shown in figure 3. It may be seen by comparison with (I) of figure 1 that extensive sulfur vulcanization is accompanied by a reduction in intensity of all of the bands of natural rubber and no new bands appear. In our previous work [1] we reported that several new bands appear in the far infrared spectrum of sulfur-vulcanized rubber. It now appears that such is not the case with either high or low sulfur content. The bands observed earlier were probably interference fringes. Interference fringes are also observed in many of the spectra presented here, especially at low wavenumbers. It might seem surprising that bands characteristic of the modified double bond structure do not appear in the far infrared spectrum as they do in the mid infrared. However, the vibrations associated with the most probable structure, trans $-\mathrm{RCH}=\mathrm{CHR}^{\prime}$, occur in the same region as the vibrations of the $\mathrm{RR}^{\prime}=\mathrm{CHR}^{\prime \prime}$ structure.

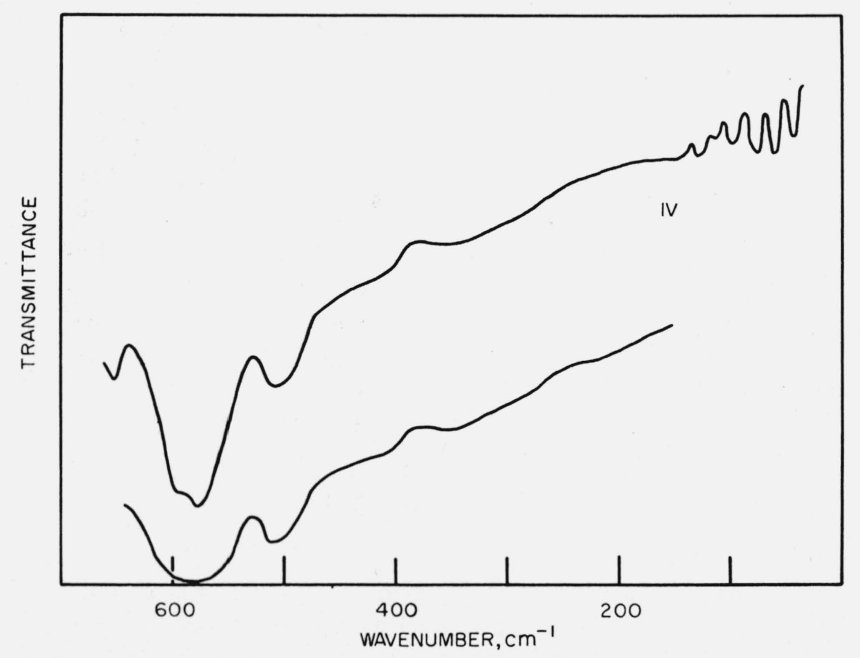

FIGURE 3. Far infrared spectrum of rubber vulcanized with sulfur (IV).

Film thickness for upper curve is about $0.2 \mathrm{~mm}$; lower curve about $0.6 \mathrm{~mm}$. 
Squalene (hexaisoprene) resembles natural rubber structurally and chemically, and the infrared spectrum of squalene in the rocksalt region is very much like that of rubber because of the similarity of functional groups. Moreover, the rocksalt infrared spectrum of the reaction product formed by squalene and sulfur resembles the spectrum of vulcanized rubber. We have extended the spectrum of squalene into the far infrared. Except for a strong squalene band at $453 \mathrm{~cm}^{-1}$ and the lack of absorption at $120 \mathrm{~cm}^{-1}$ the resemblance between the squalene and the natural rubber spectra continues into the far infrared. The band at $453 \mathrm{~cm}^{-1}$ is very likely associated with the $\left(\mathrm{CH}_{3}\right)_{2} \mathrm{C}=\mathrm{C}$ end groups of squalene. The far infrared spectrum of squalene, reacted with more than the usual amount of sulfur for $18 \mathrm{hr}$ at $150{ }^{\circ} \mathrm{C}$, shows a general reduction in intensity of all of the squalene bands and, just as in the case of the rubber vulcanizate, no new bands appear.

\section{Accelerated Sulfur Vulcanizates}

Two compounds frequently used as accelerators in sulfur vulcanization of rubber are TMTD and ZnDBDC. The far infrared spectra of natural rubber vulcanized with sulfur and TMTD and with sulfur and ZnDBDC are shown in figure 4 . The absorption bands of natural rubber are reduced in intensity just as in the case of vulcanization with sulfur alone. However, new bands are produced which are related to reaction products of the accelerators.

The spectra of TMTD, ZnDBDC, and the related compound ZnDMDC were recorded. The spectra all have absorption bands near $575 \pm 9,446 \pm 4,391 \pm 8$, $257 \pm 23 \mathrm{~cm}^{-1}$. It is likely that these vibrations are associated with the thiocarbamate structure

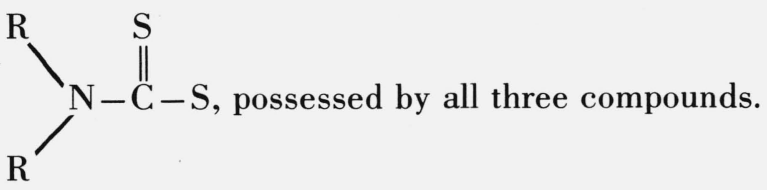

The far infrared spectrum of natural rubber highly vulcanized with sulfur and TMTD (V) has new bands at 448,395 , and $307 \mathrm{~cm}^{-1}$ and a change in the contour of the $500 \mathrm{~cm}^{-1}$ band which are not seen in the sulfur vulcanizate curve. Two of these bands are close to bands of TMTD at 444 and $398 \mathrm{~cm}^{-1}$ but TMTD has a strong band at $279 \mathrm{~cm}^{-1}$ and none at $307 \mathrm{~cm}^{-1}$. Thus the absence of a strong band at $279 \mathrm{~cm}^{-1}$ and the presence of the band at $307 \mathrm{~cm}^{-1}$ in the spectrum of $\mathrm{V}$ indicate that the bands present at 448,395, and 307 $\mathrm{cm}^{-1}$ are probably not due to a significant amount of TMTD. However, they do fall not far from the region where similar compounds absorb. Hence, it is reasonable to suppose that they are associated with a thiocarbamate structure formed during the sulfurTMTD vulcanization process.

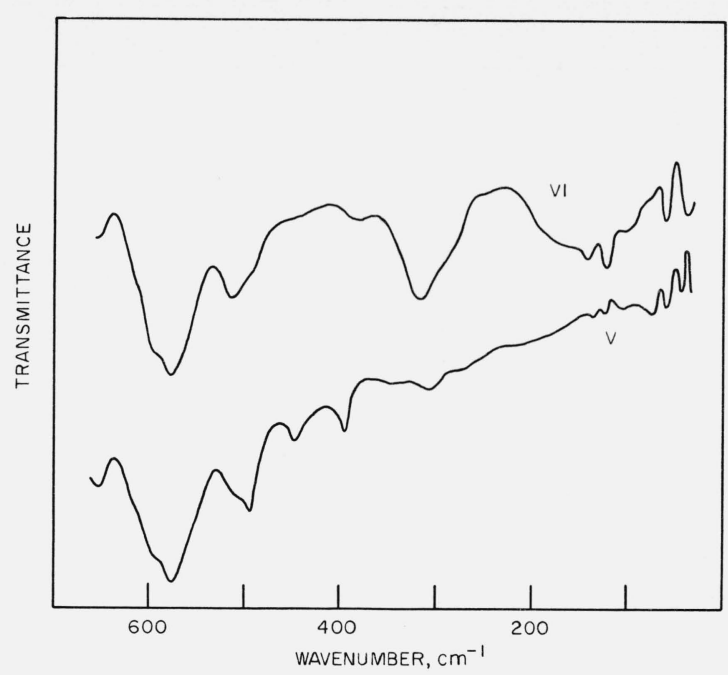

FIGURE 4. Far infrared spectra of rubber vulcanized with $(V)$ sulfur and TMTD and (VI) sulfur and ZnDBDC.

The spectrum of natural rubber highly vulcanized with sulfur and ZnDBDC (VI) has a new strong band at $310 \mathrm{~cm}^{-1}$ and another weaker broad absorption at about $150 \mathrm{~cm}^{-1}$. Notice that increased absorpion at $310 \mathrm{~cm}^{-1}$ is already evident with only 0.5 percent ZnDBDC and 1.9 percent sulfur (II) in the spectrum of figure 1. The origin of these bands is not known with certainty, but we have found that the absorption spectrum of zinc sulfide in a mineral oil mull has a strong band at about $310 \mathrm{~cm}^{-1}$.

\section{TMTD Vulcanizates}

The far infrared spectra of natural rubber vulcanized with TMTD alone (VII) is given in figure 5 . Aside from small changes in band contours, the only new feature seen in the spectrum of VII is a weak band at $302 \mathrm{~cm}^{-1}$. The spectrum of a sample identical in composition to VII, but vulcanized for only $20 \mathrm{hr}$, did not differ significantly from the spectrum of VII.

When rubber is vulcanized with TMTD in the presence of zinc oxide and stearic acid, the spectra shown in figure 6 are quite different from that shown in figure 5. TMTD is known to react with zinc oxide during vulcanization to form ZnDMDC [9]. After vulcanization for a short period there is a considerable concentration of residual TMTD and only slight evidence of the formation of ZnDMDC. The conversion continues as vulcanization progresses for longer periods of time.

The spectrum of a compound vulcanized for $45 \mathrm{~min}$ (VIII) has plainly visible TMTD bands at 86, 279 (with a shoulder at 315), 362, 399, and 444 $\mathrm{cm}^{-1}$. The band at $234 \mathrm{~cm}^{-1}$ is due to $\mathrm{ZnDMDC}$. Zinc oxide itself has a strong broad absorption at 450 $\mathrm{cm}^{-1}$ which accounts for some of the absorption in that region. At the end of $20 \mathrm{hr}$ of vulcanization (IX) 


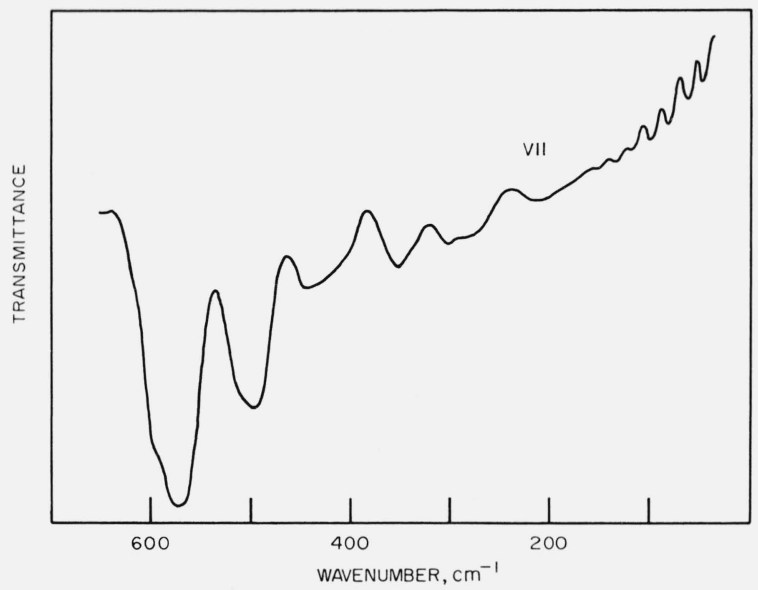

FIGURE 5. Far in frared spectrum of rubber vulcanized with TMTD $(V I I)$

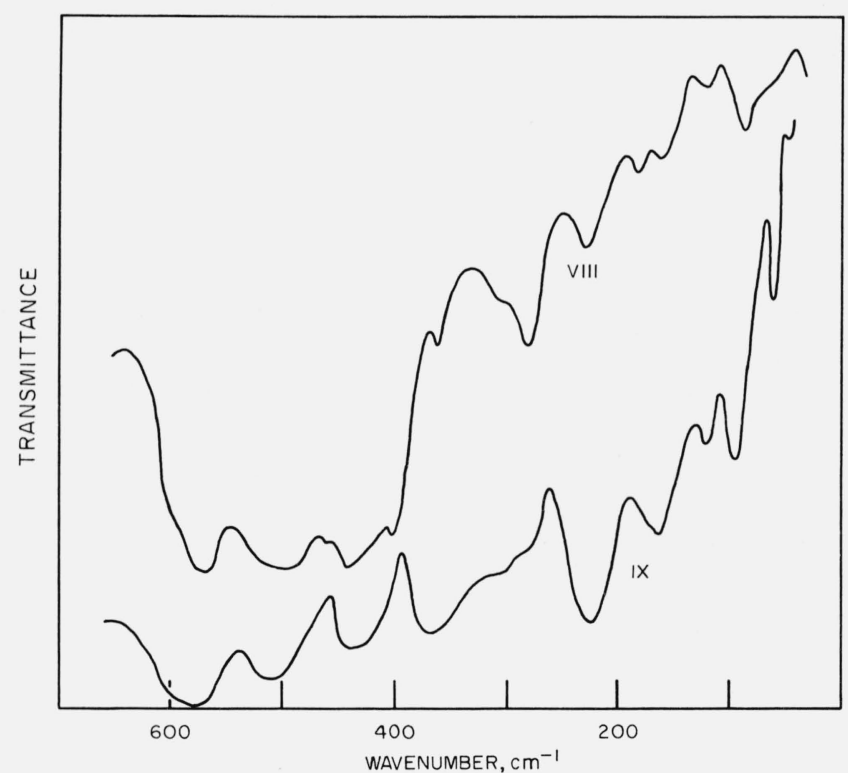

FIGURE 6. Far infrared spectra of rubber vulcanized with TMTD, zinc oxide, and st earic acid for (VIII) $45 \mathrm{~min}$, and (IX) $20 \mathrm{hr}$.

the TMTD has been nearly consumed, with only a weak absorption left at $280 \mathrm{~cm}^{-1}$, while pronounced ZnDMDC bands are found at 48, 6.1.5, 99, 120, 169, 234,385 , and $450 \mathrm{~cm}^{-1}$. Disappearance of TMTD absorptions during the course of vulcanization was also observed in the rocksalt region [1].

Aside from an increased and sharpened absorption at $445 \mathrm{~cm}^{-1}$, the spectrum of rubber vulcanized with less TMTD, zinc oxide, and stearic acid (III, fig. 1) does not display either TMTD or ZnDMDC absorption bands. Rather, it has weak bands at 407 and 462 $\mathrm{cm}^{-1}$ which do not correspond to any bands seen in the spectra of VIII or IX.

\section{Conclusion}

Low frequency vibrations of natural rubber are observed in the far infrared spectrum at 590, 570, $510,490,430,360,295,220$, and $120 \mathrm{~cm}^{-1}$. Polarized infrared studies of stretched samples show the vibrations at 570 and $295 \mathrm{~cm}^{-1}$ to be predominantly parallel to the direction of stretch and vibrations at 490 and $360 \mathrm{~cm}^{-1}$ to be perpendicular. These polarization sensitive vibrations occur in the crystalline portion of the rubber. The vibrations at 590 and $510 \mathrm{~cm}^{-1}$ originate in the amorphous portion and are insensitive to polarization.

The effect of vulcanization on the far infrared spectrum of natural rubber can be summarized as follows:

(1) Vulcanization with sulfur alone produces no new bands, but an overall weakening of the absorption bands of natural rubber.

(2) Vulcanization with sulfur and an accelerator reduces the intensity of the rubber absorptions and introduces new bands, some of which have been tentatively explained. When the accelerator is TMTD new bands at $458,395,307$ and perhaps near $500 \mathrm{~cm}^{-1}$ probably originate in thiocarbamate structures. When $\mathrm{ZnDBDC}$ is used as an accelerator new bands are found at 310 and $150 \mathrm{~cm}^{-1}$. The former might be due to zinc sulfide.

(3) Vulcanization with TMTD in the absence of elemental sulfur and zinc oxide introduces only minor changes in band shapes and a weak band at $302 \mathrm{~cm}^{-1}$.

(4) After brief vulcanization with TMTD in the presence of zinc oxide and stearic acid, bands contributed by unreacted TMTD at $86,279,315,362$, 399 , and $444 \mathrm{~cm}^{-1}$ and zinc oxide at $450 \mathrm{~cm}^{-1}$ remain. If the vulcanization is continued for a longer period the reaction of TMTD with zinc oxide produces ZnDMDC which contributes bands at $48,61.5,99$, $120,169,234,385$, and $450 \mathrm{~cm}^{-1}$.

\section{References}

[1] F. J. Linnig and J. E. Stewart, J. Res. NBS 60, 9 (1958) RP2816; Rubber Chem. Technol. 31, 719 (1958).

[2] F. J. Linnig, E. J. Parks, and J. E. Stewart, J. Res. NBS 68A (Phys. and Chem.) No. 4, 499 (1964).

[3] G. B. B. M. Sutherland and A. V. Jones, Disc. Faraday Soc. 9, 281 (1950).

[4] R. A. Saunders and D. C. Smith, J. Appl. Phys. 20, 953 (1949).

[5] J. E. Stewart, Far Infrared Spectroscopy, Interpretive Spectroscopy, S. K. Freeman, ed. (Rheinhold, New York, 1965).

[6] J. J. Shipman and M. A. Golub, J. Polymer Sci. 58, 1063 (1962).

[7] T. Miyazawa, Y. Ideguchi, and K. Fukushima, J. Chem. Phys. 38, 2709 (1963).

[8] H. Tadokoro, M. Kobayashi, M. Ukita, K. Yasufuku, S. Murahashi, and T. Torii, J. Chem. Phys. 42, 1432 (1965).

[9] W. Scheele, O. Lorenz, and W. Dummer, Kautshuk u. Gummi 7, WT 273 (1954); Rubber Chem. Technol. 29, 1 (1956).

(Paper 71Al-432) 